Desde las aulas

\title{
Efecto de una barra alimenticia sobre los niveles séricos de glucosa
}

\section{Nutritional bar effects over glucose serum levels}

\author{
González-Martínez, María Fernanda; González-Martínez, Karla \\ Paola; González-Cabello, María Dolores; Plascencia-Esquivias, \\ Yatziry Yahel; Chávez-Esparza, Alexandra; Ramírez-Herna, Laura \\ Alejandra; Martín del Campo-Cervantes, Judith
}

\author{
María Fernanda González-Martínez \\ Universidad Autónoma de Aguascalientes, México \\ Karla Paola González-Martínez \\ Universidad Autónoma de Aguascalientes, México \\ María Dolores González-Cabello \\ Universidad Autónoma de Aguascalientes, México \\ Yatziry Yahel Plascencia-Esquivias \\ Universidad Autónoma de Aguascalientes, México \\ Alexandra Chávez-Esparza \\ Universidad Autónoma de Aguascalientes, México \\ Laura Alejandra Ramírez-Herna \\ Universidad Autónoma de Aguascalientes, México \\ Judith Martín del Campo-Cervantes \\ judith.martindelcampo@edu.uaa.mx \\ Universidad Autónoma de Aguascalientes, México
}

\author{
Lux Médica \\ Universidad Autónoma de Aguascalientes, México \\ ISSN: 2007-1655 \\ Periodicidad: Cuatrimestral \\ vol. 17, núm. 49, 2022 \\ luxmedica.editorial@gmail.com \\ Recepción: 13 Octubre 2021 \\ Aprobación: 08 Noviembre 2021 \\ URL: \\ https://revistas.uaa.mx/index.php/luxmedica/article/view/3376 \\ DOI: \\ https://doi.org/10.33064/491m20223376
}

Resumen: Introducción: La diabetes mellitus tipo dos es un problema de salud a nivel mundial. En México, el 10.6\% de la población presenta diabetes, y en los últimos cinco años ha sido la segunda causa de muerte en el estado de Aguascalientes. La avena y el salvado de trigo son una fuente fibra con un índice glucémico bajo; los arándanos y las almendras pueden tener un efecto benéfico en los niveles de glucosa sérica. Objetivo: Evaluar la variación de los niveles séricos de glucosa después del consumo de una barra nutricional. Material y métodos: Se estudiaron 32 participantes los cuales se dividieron en dos grupos, un grupo consumió una barra de avena y otro una barra de salvado de trigo; se evaluaron los niveles de glucemia en ayuno y postprandial de dos horas. Resultados: El promedio de la glucosa postprandial del grupo que consumió la barra de salvado de trigo $(90.43 \mathrm{mg} /$ $\mathrm{dL} \pm 7.08 \mathrm{mg} / \mathrm{dL}$ ) fue menor que el presentado por el grupo que consumió la barra de avena $(95.37 \mathrm{mg} / \mathrm{dL} \pm 9.09 \mathrm{mg} / \mathrm{dL})$, sin encontrar diferencias significativas entre barras $(t=1.713$, $\mathrm{p}=0.097$ ). Conclusión: El consumo de una barra nutricional (avena o salvado de trigo) no provoca un aumento de glucosa sérica postprandial significativo. Se puede recomendar el consumo de estas barras nutricionales en personas que padecen diabetes de acuerdo a estos resultados.

Palabras clave: diabetes mellitus, barra nutricional, avena, salvado de trigo, hiperglucemia.

Abstract: Introduction: Type two diabetes is a worldwide health problem, in Mexico 10.6\% of the population has diabetes and in the last five years it has been the second cause of death in the state of Aguascalientes. On the other hand, food like oatmeal and wheat bran are a low-glycemic index fiber source, cranberries and almonds may also be beneficial for serum glucose levels. Objective: To evaluate the variation of serum glucose levels after the consumption of a nutritional bar. Material and methods: 32 participants were studied, they were divided into two groups, one group consumed an oatmeal bar and the other one a wheat bran bar, fasting and postprandial (two hours) blood glucose levels were evaluated. Results: The average postprandial glucose of the group that consumed the wheat bran bar $(90.43 \mathrm{mg} / \mathrm{dL} \pm 7.08$ $\mathrm{mg} / \mathrm{dL}$ ) was lower than the one presented by the group that consumed the oatmeal bar $(95.37 \mathrm{mg} / \mathrm{dL} \pm 9.09 \mathrm{mg} / \mathrm{dL})$, 
without finding significant differences between bars $(t=1.713$, $p=0.097)$. Conclusion: The consumption of a nutritional bar (oatmeal or wheat bran) doesn't cause a significant postprandial increase in serum glucose. The consumption of this nutritional bars can be recommended in people with diabetes according with these results.

Keywords: type two diabetes, nutritional bar, oats, wheat bran, hyperglycemia.

\section{INTRODUCCIÓN}

De acuerdo con la Asociación Americana para la Diabetes, la diabetes mellitus (DM) "es un grupo de trastornos metabólicos caracterizados por la hiperglucemia resultante de los defectos de la secreción o la acción de la insulina, o ambas". En específico, la diabetes mellitus tipo 2 (DMT2) es ocasionada por la deficiencia de insulina como consecuencia de la falla o disminución de células $\beta$ del páncreas, o una resistencia a la insulina ostentada por una mayor producción de glucosa, provocando una hipoglucemia intracelular o una hiperglucemia extracelular. ${ }^{2,3}$ La hiperglucemia es definida como el aumento de la glucosa sérica ocasionada por los cambios metabólicos y hormonales asociados con el aumento de las hormonas circulantes contrarreguladoras inflamatorias que interfieren con el metabolismo de los hidratos de carbono. ${ }^{4,5}$

La DMT2 es considerada un problema de salud pública no solo en México sino también a nivel mundial. La Encuesta Nacional de Salud y Nutrición 2020 (ENSANUT) estimó que la prevalencia de esta enfermedad en México es del $10.6 \% .{ }^{6}$ Se estima que esta patología reduce entre cinco y diez años la esperanza de vida de la población. ${ }^{7}$

Una alimentación correcta (completa, equilibrada, suficiente, inocua, adecuada y variada) representa un pilar fundamental en el tratamiento de la DMT2. La terapéutica nutricional aplicada al tratamiento de la DMT2 considera alimentos de bajo índice glucémico (IG). Este categoriza a los alimentos que contienen hidratos de carbono en relación con su capacidad de incrementar los niveles de glucemia (velocidad y magnitud), ${ }^{8}$ con lo cual se busca disminuir la absorción intestinal de los hidratos de carbono y, por lo tanto, los niveles de glucosa que se encontrarán en el torrente sanguíneo en periodo pospandrial. ${ }^{9}$

Los cereales integrales son una fuente común de fibra que además tienen un IG bajo, ${ }^{10}$ como es el caso de la avena, por su contenido de $\beta$-glucanos y almidón resistente, ${ }^{11}$ y del salvado del trigo, por el contenido de arabinoxilanos. ${ }^{12}$ Estos componentes tienen efectos directos en la regulación del metabolismo de los hidratos de carbono, ${ }^{11,13}$ lo cual justifica su uso como alimentos funcionales en la dieta de las personas que padecen DMT2. Los arándanos ${ }^{14}$ y las almendras ${ }^{15}$ son considerados alimentos funcionales que pueden tener un efecto benéfico en el control glucémico en pacientes que padecen diabetes por su contenido de proteína vegetal, ácido oleico, calcio, vitamina E, hierro y magnesio. ${ }^{15}$ 
$\mathrm{Al}$ combinar los alimentos anteriormente descritos en forma de una barra de cereal se espera que la velocidad de absorción a nivel intestinal de los hidratos de carbono disminuya y tenga un efecto favorable en las cifras de glucosa sérica.

Se deben buscar alternativas de snacks o alimentos que coadyuven con el tratamiento médico y la terapia nutricional para mantener los niveles de glucemia en niveles deseables en personas que padezcan alteraciones en el metabolismo de la glucosa, como es el caso de la DMT2. El objetivo de la presente investigación fue evaluar la variación de los niveles séricos de glucosa después del consumo de una barra nutricional.

\section{MATERIALES Y MÉTODOS}

Diseño. Ensayo clínico comparativo de corte transversal. Participantes: se seleccionó una muestra total de 32 adultos mayores de 18 años de edad y menores de 65 años de edad (23 mujeres y nueve hombres); residentes de la ciudad de Aguascalientes, México; sin presencia de enfermedades crónico degenerativas; con un índice de masa corporal (IMC) de entre 18.5 y $29.9 \mathrm{~kg} / \mathrm{m}^{2}$. Fueron excluidos los individuos con diagnóstico establecido de DMT2, síndrome de ovario poliquístico, obesidad, hipertensión o enfermedad tiroidea, así como aquellos que presentaron glucosa sérica en ayunas $>126 \mathrm{mg} / \mathrm{dL}$, individuos en tratamiento hormonal, mujeres embarazadas y personas con alergia al trigo, avena, almendra, arándano y huevo. Todo participante firmó una carta de consentimiento informado. El proyecto estuvo apegado a la declaración de Helsinki y al Reglamento de Investigación de la Ley General de Salud.

Plan de trabajo. Se evaluaron los niveles de glucosa capilar con los glucómetros marca SD check, ACCU-CHEK Performa, ACCU-CHEK Active y OneTouch Ultra. Barra: Se elaboraron dos barras nutricionales. La primera a base de $20 \mathrm{~g}$ de avena, $2 \mathrm{~g}$ arándanos secos, $3 \mathrm{~g}$ de almendra fileteada con cáscara y $12 \mathrm{~g}$ de clara de huevo como aglutinante. La segunda barra se preparó con $20 \mathrm{~g}$ de salvado de trigo, $2 \mathrm{~g}$ de arándanos secos, $4 \mathrm{~g}$ de almendra fileteada con cáscara y $24 \mathrm{~g}$ de clara de huevo como aglutinante. Para la barra de salvado de trigo se licuó el salvado de trigo junto con los arándanos por 45 segundos, se le agregaron los demás ingredientes y se mezclaron; posteriormente, se le dio forma a la barra y se colocó en el horno a una temperatura de $180^{\circ} \mathrm{C}$ por 20 minutos. La preparación de la barra de avena fue la siguiente: Se licuó la avena integral junto con los arándanos por 45 segundos; posteriormente, se agregaron los demás ingredientes y se mezclaron, se le dio forma a la barra y se colocó en el horno a una temperatura de $180^{\circ} \mathrm{C}$ por 20 minutos.

Ambas barras tienen una distribución similar de macronutrientes (tabla 1). El peso neto de la barra de avena fue de $35 \mathrm{~g}$ por ración y para la barra de salvado de trigo fue de $45 \mathrm{~g}$ por ración. 
Tabla 1. Cantidades de macronutrientes y calorías por porción

\begin{tabular}{lllll}
\multicolumn{5}{c}{ Tabla 1. Cantidades de macronutrientes y calorias por porción } \\
\hline Tipo de barra & $\begin{array}{l}\text { Hidratos } \\
\text { de } \\
\text { carbono } \\
\text { (g) }\end{array}$ & $\begin{array}{l}\text { Lipidos } \\
(\mathrm{g})\end{array}$ & $\begin{array}{l}\text { Proteinas } \\
\text { (g) }\end{array}$ & $\begin{array}{l}\text { Energía } \\
\text { (Kcal) }\end{array}$ \\
\hline $\begin{array}{l}\text { Barra de avena } \\
\text { Barra de salvado } \\
\text { de trigo }\end{array}$ & 21 & 1 & 4 & 119 \\
\hline
\end{tabular}

Fuente: realización propia.

Se formaron dos grupos de forma aleatoria simple mediante tómbola. A un grupo se le brindó la barra nutricional de avena y al otro la barra nutricional de salvado de trigo. Se realizaron dos tomas de glucosa capilar a cada individuo previo ayuno de al menos ocho horas. La primera medición fue en ayunas y la segunda medición fue postprandial, dos horas después del consumo de la barra nutricional y sin que los participantes consumieran otros alimentos ni líquidos en ese periodo.

Análisis estadístico: se aplicó estadística inferencial mediante el uso del programa SPSS/PC versión 25 para Windows. Se utilizó la prueba t de student para muestras relacionadas, y la prueba de normalidad Shapiro-Wilk. El valor de $p<0.05$ fue asumido para significancia estadística con un $95 \%$ de nivel de confianza.

\section{RESULTADOS}

De la población estudiada, el $71.9 \%$ fueron mujeres $(n=23)$ y el $28.10 \%$ fueron hombres $(n=9)$. La edad promedio de la población fue de 31.65 años de edad (DE \pm 14.63 años de edad). Los demás datos se encuentran descritos en la tabla 2 .

Tabla 2. Características descriptivas de la población. Promedio y desviación estándar

\begin{tabular}{lllllll}
\hline $\begin{array}{l}\text { Tipo de } \\
\text { barra }\end{array}$ & Hombres & Mujeres & $\begin{array}{l}\text { IMC } \\
(\mathrm{Kg} / \mathrm{m} 2)\end{array}$ & Peso $(\mathrm{Kg})$ & $\begin{array}{l}\text { Estatura } \\
(\mathrm{m})\end{array}$ & Edad (años) \\
\hline $\begin{array}{l}\text { Barra de } \\
\text { avena }\end{array}$ & 5 & 11 & $26.24 \pm 2.6$ & $69.4 \pm 9.3$ & $1.61 \pm 0.09$ & $34 \pm 15.3$ \\
$\begin{array}{l}\text { Barra de } \\
\text { salvado de } \\
\text { trigo }\end{array}$ & 4 & 12 & $23.7 \pm 3.1$ & $66.4 \pm 14.3$ & $1.66 \pm 0.08$ & $29.25 \pm 13.9$ \\
\hline
\end{tabular}

Datos representados como media y desviación estándar. $(\mathrm{n}=32)$ participantes (16 en cada grupo); IMC: índice de masa corporal.

El índice de masa corporal presentó un puntaje promedio poblacional de 25.0 $\mathrm{kg} / \mathrm{m}^{2}\left(\mathrm{DE} \pm 3.23 \mathrm{~kg} / \mathrm{m}^{2}\right)$. Al clasificarlo se encontró que el $40.6 \%$ de la muestra presentó normopeso $(\mathrm{n}=13)$ y el $59.4 \%$ de los participantes presentó sobrepeso $(\mathrm{n}=19)$.

Al 50\% de los participantes se le proporcionó una barra de avena y al otro $50 \%$ se le proporcionó una barra de salvado de trigo previamente elaborada con las recetas estandarizadas mencionadas anteriormente. Posteriormente, se realizó la toma de glucosa capilar en ayunas (con ocho horas previas de ayuno) y dos 
horas después del consumo de las barras. Los niveles promedio de glucosa capilar poblacionales en ayunas fueron de $92.31 \mathrm{mg} / \mathrm{dL}( \pm 8.90 \mathrm{mg} / \mathrm{dL})$ y los niveles postprandiales fueron de $92.90 \mathrm{mg} / \mathrm{dL}( \pm 8.40 \mathrm{mg} / \mathrm{dL})$.

El promedio de la glucosa postprandial (figura 1) del grupo que consumió la barra de salvado de trigo fue menor $(90.43 \mathrm{mg} / \mathrm{dL})$ que el presentado por el grupo que consumió la barra de avena $(95.37 \mathrm{mg} / \mathrm{dL})$. Dicha diferencia no fue estadísticamente significativa (tabla 3 ), por lo que la glucosa no presentó una modificación independientemente del tipo de barra consumida $(t=1.713$, $\mathrm{p}=0.097)$.

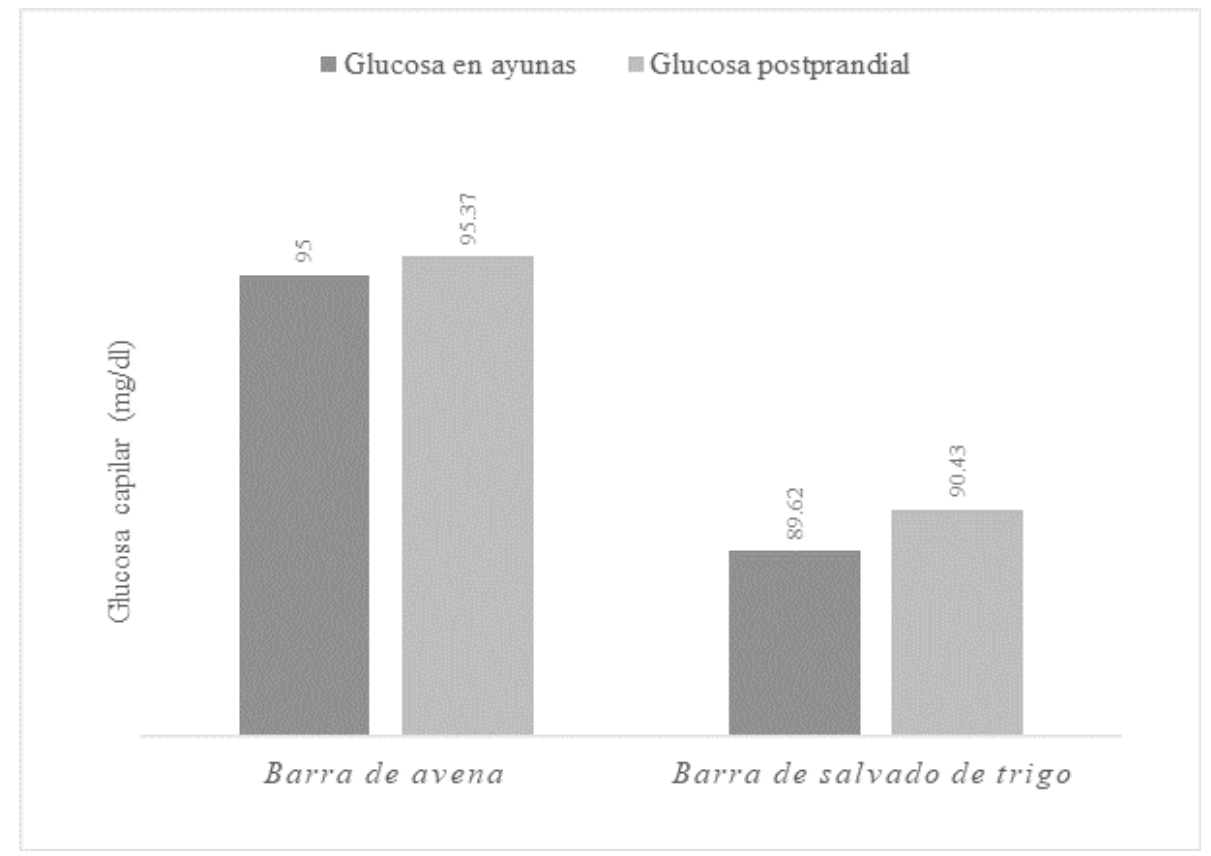

Figura 1. Promedio de glucosa en ayunas y pospandrial por grupo de estudio

Tabla 3. Glucosa capilar postprandial por grupo de estudio

\begin{tabular}{llll}
\hline Analito & $\begin{array}{c}\text { Promedio (DE) grupo } \\
\text { barra de avena }\end{array}$ & $\begin{array}{l}\text { Promedio (DE) grupo } \\
\text { barra de salvado de trigo }\end{array}$ & $\mathrm{p}$ \\
\hline Glucosa capilar (mg/dL) & $95.37 \pm 9.09$ & $90.43 \pm 7.08$ & 0.097 \\
\hline
\end{tabular}

Datos representados como media y desviación estándar. Valores de $\mathrm{p}$ utilizando la prueba $\mathrm{t}$ de student para muestra no relacionadas, $\mathrm{p}<0.05 . \mathrm{n}=32$ participantes ( 16 para cada grupo).

No se encontró una diferencia entre la glucosa en ayunas y postprandial en los participantes que consumieron la barra de avena $(\mathrm{t}=-0.212, \mathrm{p}=0.835)$ y la barra de salvado de trigo $(t=-0.345, p=0.735)$, lo que indica que el tipo de fibra presente en cada barra nutricional no genera una modificación en las cifras de glucemia capilar (tabla 4). 
Tabla 4. Glucosa capilar en ayunas y postprandial por tipo de barra

\begin{tabular}{llll}
\hline Tipo de barra & $\begin{array}{l}\text { Glucosa capilar en } \\
\text { ayunas (mg/dL) Media (DE) }\end{array}$ & $\begin{array}{l}\text { Glucosa capilar postprandial } \\
(\mathrm{mg} / \mathrm{dL}) \text { Media }(\mathrm{DE})\end{array}$ & $\mathrm{p}$ \\
\hline Barra de avena & $95.00 \pm 8.85$ & $95.37 \pm 9.09$ & 0.835 \\
Barra de salvado de trigo & $89.62 \pm 8.37$ & $90.43 \pm 7.08$ & 0.735 \\
\hline
\end{tabular}

Datos representados como media y desviación estándar. $n=32$ participantes ( 16 para cada grupo). Valores de $\mathrm{p}$ utilizando la prueba t de student para muestra relacionadas, $\mathrm{p}<0.05$.

\section{DISCUSIÓN}

El estudio de Sun y $\operatorname{Cols}^{16}$ indica que la avena reduce el nivel de glucosa capilar postprandial por la acción de los $\beta$-glucanos, al producir un efecto hipoglucemiante, favorecer una gradual absorción de glucosa y mejorar la sensibilidad a la insulina a través del retraso del vaciamiento gástrico secundario a la alta viscosidad y estabilidad del $\mathrm{pH}$ de la avena. Los $\beta$-glucanos tienen, además, la capacidad de unirse con las grasas provenientes de la dieta, lo cual trae como efecto benéfico la disminución en la absorción no solo de glucosa, sino también de las grasas. ${ }^{17}$ Los resultados obtenidos en la población estudiada indican que, al incluir avena en la barra nutricional, probablemente ocurrieron los mismos efectos. Por lo tanto, no se obtuvieron cambios significativos en las concentraciones de glucosa capilar postprandial. ${ }^{16}$

El estudio de Arun y $\mathrm{Cols}^{17}$ menciona que el salvado de trigo posee fitoquímicos -como kaempferol, apigenina, catecol, ácido p-cumarico, ácido ferúlico, entre otros-, los cuales, al inhibir la $\alpha$-amilasa y $\alpha$-glucosidasa, mejoran la absorción de glucosa e inhiben a las enzimas claves involucradas en el metabolismo de los hidratos de carbono, lo que causa la reducción de la hiperglucemia postprandial. ${ }^{17}$ La barra nutricional elaborada con salvado de trigo presentó resultados parecidos a los encontrados por Arun y Cols.

Otro de los ingredientes utilizados en la elaboración de las barras fue la almendra fileteada con cáscara. El estudio de Barreca y Cols ${ }^{18}$ la asocia con una mayor sensibilidad a la insulina postprandial y una disminución de los niveles de glucosa postprandial por su contenido de grasa, proteína y fibra. La almendra por su contenido de polifenoles, específicamente de los flavonoides, reduce el daño proteico inducido por el estrés oxidativo que ocurre como consecuencia de la hiperglucemia prolongada o por el retraso del vaciamiento gástrico. De acuerdo a los resultados obtenidos con las dos versiones de barras nutricionales, se genera mayor evidencia a favor del uso de almendras como alimento funcional para el control de la glucemia. ${ }^{18}$

El estudio de Schell y Cols ${ }^{19}$ reportó que los arándanos secos se asocian con una disminución significativa en la glucosa sérica en ayunas y postprandial. Las proantocianidinas, que son los principales polifenoles de los arándanos, actúan en la inhibición de las enzimas digestivas de los hidratos de carbono y en la mejora de la captación y el metabolismo de la glucosa e inhibición de los transportadores intestinales de la misma, por lo cual reducen la absorción de la glucosa en la 
circulación. ${ }^{19}$ Por lo anterior, se consideró incluir arándanos en la receta de las barras nutricionales.

Los componentes que conforman la barra nutricional son alimentos que tienen efectos benéficos en los niveles de glucosa sérica. Además, el contenido de hidratos de carbono $(15 \mathrm{~g})$ de las barras es reducido en comparación con versiones comerciales; es por esto que ambas barras pueden ser consideradas como parte de la alimentación de una persona sana o con diabetes mellitus tipo 2.

Los investigadores declaran no tener conflictos de interés en la realización de la investigación. Las limitaciones del estudio fueron la utilización de diferentes instrumentos de medición (glucómetros). Debido a la contingencia sanitaria a causa de la SARS-CoV-2 (COVID-19), se modificó la forma de obtención de los participantes y la medición de las glucemias. Al tener estas limitaciones se considera que pudo haber variabilidad en los resultados obtenidos por cada uno de los investigadores.

\section{CONCLUSIÓN}

El consumo de una barra nutricional (avena o salvado de trigo) no produce un aumento de glucosa sérica postprandial significativo.

Con base en lo anterior, se proponen futuras investigaciones en el uso de este tipo de barras nutricionales en personas que padecen diabetes y las alteraciones que pueden provocar en el nivel de glucosa sérica.

\section{Conflicto de intereses}

Sin conflicto de intereses.

\section{Referencias}

1. American Diabetes Association. Classification and Diagnosis of Diabetes: Standards of Medical Care in Diabetes. Diabetes Care. 2020; 43 (Supplement 1): S14-S31. https://doi.org/10.2337/dc20-S002.

2. Pinés Corrales PJ, Bellido Castañeda V, Ampudia-Blasco FJ. Actualización sobre hiperglucemia posprandial: fisiopatología, prevalencia, consecuencias e implicaciones para el tratamiento de la diabetes. Rev. Clin (Esp). 2020; 220(1):57-68. doi: 10.1016/j.rce.2018.06.015.

3. Ullah A, Khan A, Khan I. Diabetes mellitus and oxidative stress - A concise review. Saudi Pharm J. 2016; 24(5):47-553. doi: 10.1016/j.jsps.2015.03.013.

4. Umpierrez GE, Pasquel FJ. Management of Inpatient Hyperglycemia and Diabetes in Older Adults. Diabetes Care. 2017; 40(4):509-517. doi: 10.2337/dc16-0989.

5. Davies MJ, D'Alessio DA, Fradkin J, Kernan WN, Mathieu C, Mingrone G, Rossing P, Tsapas A, Wexler DJ, Buse JB. Management of Hyperglycemia in Type 2 Diabetes, 2018. A Consensus Report by the American Diabetes Association (ADA) and the European Association for the Study of Diabetes (EASD). Diabetes Care. 2018; 41(12):2669-2701. doi: 10.2337/dci18-0033.

6. Shamah-Levy T, Romero-Martínez M, Barrientos-Gutiérrez T, Cuevas-Nasu L, Bautista-Arredondo S, Colchero MA, GaonaPineda EB, Lazcano-Ponce E, Martínez-Barnetche J, Alpuche-Arana C, Rivera-Dommarco J. Encuesta 
Nacional de Salud y Nutrición 2020 sobre Covid-19. Resultados nacionales. Cuernavaca, México: Instituto Nacional de Salud Pública, 2021. Disponible en: https://ensanut.insp.mx/encuestas/ensanutcontinua2020/doctos/informes/ ensanutCovid19ResultadosNacionales.pdf

7. Gutiérrez JP, Rivera-Dommarco J, Shamah-Levy T, Romero-Martínez M, Hernández-Ávila M. Encuesta Nacional de Salud y Nutrición 2017. Resultados nacionales. 2a. ed. Cuernavaca, México: Instituto Nacional de Salud Pública, 2017.

8. Morales Guerrero J. C, Rosas Romero R, García Zepeda R. A, Oropeza Hernández R, Ruiz Jiménez S, Ríos Chávez A. et al. Determinación del índice glucémico y la carga glucémica de productos lácteos fermentados en sujetos adultos sanos, sedentarios y deportistas. Nutr. Hosp. 2016; 33(5):1095-1101.

9. American Diabetes Association. Prevention or Delay of Type 2 Diabetes: Standards of Medical Care in Diabetes-2020. Diabetes Care. 2020 Jan; 43(Suppl 1):S32S36. doi: $10.2337 / \mathrm{dc} 20-S 003$.

10. Pérez-Cruz E, Calderón-Du Pont D, Cardoso-Martínez C, Dina-Arredondo V, et al. Estrategias nutricionales en el tratamiento del paciente con diabetes mellitus. Rev Med Inst Mex Seguro Soc. 2020; 58(1):50-60.

11. Yingying Z, Lianger D, Lu H, Zhenxing S, Jilin D, Yang Y, Ruiling S. Effects of oat $\beta$-glucan, oat resistant starch, and the whole oat flour on insulin resistance, inflammation, and gut microbiota in high-fat-diet-induced type 2 diabetic rats. J Funct Foods. 2020; 69:103939. doi: 10.1016/j.jff.2020.103939.

12. Mathers J. Choosing your carbohydrates to prevent DM. Br J Nutr. 2002; 88(2):107-108. doi: 10.1079/BJNBJN2002606.

13. Kjølbæk L, Benítez-Páez A, Gómez Del Pulgar EM, Brahe LK, Liebisch G, Matysik S, Rampelli S, Vermeiren J, Brigidi P, Larsen LH, Astrup A, Sanz Y. Arabinoxylan oligosaccharides and polyunsaturated fatty acid effects on gut microbiota and metabolic markers in overweight individuals with signs of metabolic syndrome: A randomized cross-over trial. Clin Nutr. 2020; 39(1):67-79. doi: 10.1016/ j.clnu.2019.01.012.

14. Huang W, Yao L, He X, et al. Hypoglycemic activity and constituents analysis of blueberry (Vaccinium corymbosum) fruit extracts. Diabetes Metab Syndr Obes. 2018; 11:357-366. doi:10.2147/DMSO.S166728

15. Li SC, Liu YH, Liu JF, Chang WH, Chen CM, Chen CY. Almond consumption improved glycemic control and lipid profiles in patients with type 2 diabetes mellitus. Metab. 2011; 60(4):474-9. doi: 10.1016/j.metabol.2010.04.009.

16. Sun $\mathrm{T}$, et al. Rheological and functional properties of oat $\beta$-glucan with different molecular weight. J Mol Struct. 2020; 1209:127944. doi: 10.1016/ j.molstruc.2020.127944

17. Arun KB, Dhanya R, Chandran J, Abraham B, Satyan S, Nisha P. A comparative study to elucidate the biological activities of crude extracts from rice bran and wheat bran in cell line models. J Food Sci Technol. 2020; 57(9):3221-3231. doi: 10.1007/s13197-020-04353-1.

18. Barreca D, Nabavi SM, Sureda A, Rasekhian M, Raciti R, Silva AS, Annunziata G, Arnone A, Tenore GC, Süntar İ, Mandalari G. Almonds (Prunus Dulcis Mill. D. A. Webb): A Source of Nutrients and Health-Promoting Compounds. Nutrients. 2020; 12(3):672. doi: 10.3390/nu12030672.

19. Schell J, Betts NM, Foster M, Scofield RH, Basu A. Cranberries improve postprandial glucose excursions in type 2 diabetes. Food Funct. 2017; 8(9):3083-3090. doi: 10.1039/c7fo00900c. 